\title{
Existence, Stationary Distribution, and Extinction of Predator-Prey System of Prey Dispersal with Stochastic Perturbation
}

\author{
Li Zu, ${ }^{1,2}$ Daqing Jiang, ${ }^{1}$ and Fuquan Jiang ${ }^{3}$ \\ ${ }^{1}$ School of Mathematics and Statistics, Northeast Normal University, Changchun 130024, China \\ ${ }^{2}$ School of Science, Changchun University, Changchun 130022, China \\ ${ }^{3}$ Department of Foundation, Harbin Finance University, Harbin 150030, China
}

Correspondence should be addressed to Daqing Jiang, daqingjiang2010@hotmail.com

Received 24 July 2012; Accepted 13 September 2012

Academic Editor: Yonghui Xia

Copyright (C) $2012 \mathrm{Li} \mathrm{Zu}$ et al. This is an open access article distributed under the Creative Commons Attribution License, which permits unrestricted use, distribution, and reproduction in any medium, provided the original work is properly cited.

We consider a predator-prey model in which the preys disperse among $n$ patches $(n \geq 2)$ with stochastic perturbation. We show that there is a unique positive solution and find out the sufficient conditions for the extinction to the system with any given positive initial value. In addition, we investigate that there exists a stationary distribution for the system and it has ergodic property. Finally, we illustrate the dynamic behavior of the system with $n=2$ via numerical simulation.

\section{Introduction}

Interest has been growing in the study of the dynamic relationship between predators and their preys due to its universal existence and importance. However, due to the spatial heterogeneity and the increasing spread of human activities, the habitats of many biological species have been separated into isolated patches. In some of these patches, without the contribution from other patches, the species will go to extinction. Recently, the effect of dispersion on the species survival has been an important subject in population biology (see [1-10] and the references cited therein). Particularly, two species predator-prey systems with dispersal have received great attention from both theoretical and mathematical biologists and many good results have been achieved (see [1, 2, 7, 9-11]). The analysis of these papers has been centered around the coexistence of populations, stability (local and global), and permanence of equilibria. Zhang and Teng [11] established the sufficient conditions on the boundedness, permanence, and existence of positive periodic solution for two species predator-prey model. Kuang and Takeuchi [1] studied a predator-prey system with prey 
dispersal in a two-patch environment; they obtained the existence, local and global stability of the positive steady state and analyzed both the stabilizing and destabilizing effects of dispersion by introducing examples.

$\mathrm{Li}$ and Shuai [2] considered the model

$$
\begin{aligned}
& \dot{x}_{i}=x_{i}\left(r_{i}-b_{i} x_{i}-e_{i} y_{i}\right)+\sum_{j=1}^{n} d_{i j}\left(x_{j}-\alpha_{i j} x_{i}\right), \\
& \dot{y}_{i}=y_{i}\left(-\gamma_{i}-\delta_{i} y_{i}+\varepsilon_{i} x_{i}\right), \quad i=1,2, \ldots, n,
\end{aligned}
$$

where $x_{i}, y_{i}$ denote the densities of preys and predators on the patch $i$, respectively. The parameters in the model are nonnegative constants and $e_{i}, \varepsilon_{i}$ are positive. The constants $d_{i j}$ are the dispersal rate from patch $j$ to $i$, and the constants $\alpha_{i j}$ can be selected to represent different boundary conditions in the continuous diffusion case [12]. Let $\left(d_{i j}\right)$ denote $n \times n$ dispersal matrix. By constructing a Lyapunov function and using graph theory, Li and Shuai proved the uniqueness and globally asymptotically stability of a positive equilibrium, whenever it exists, if $\left(d_{i j}\right)$ is irreducible and there exists $i$ such that $b_{i}>0$ or $\delta_{i}>0$.

The model mentioned above is a deterministic model which assumes that the parameters in the model are all deterministic irrespective environmental fluctuations. In fact, population dynamics is inevitably affected by environmental white noise, such as weather and epidemic disease. Therefore, the deterministic models are often subject to stochastic perturbation, and it is useful to reveal how the noise affects the population system. There are some authors who have studied the dynamics of predator-prey models with stochastic perturbations (see [13-15]). Ji et al. [13] studied a predator-prey with modified Leslie-Gower and Holling-type II schemes with stochastic perturbation; they got some good results about existence, uniqueness, and extinction of positive solution. Cai and Lin [15] investigated a predator-prey stochastic system with competition among predators and obtained the probability distribution of the system state variables at the state of statistical stationarity. But, until now, few people study the dynamical behavior of the predator-prey system with diffusion under the influence of white noise. However, the diffusion phenomenon and environmental white noise are universal existence in nature. Therefore, we want to study the effect of random perturbations on the predator-prey system on the basis of the existing diffusion model and the contents of this paper are of great significance.

In this paper, we take into account the effect of randomly fluctuating and stochastically perturb intrinsic growth rate in each equations of (1.1):

$$
\begin{gathered}
r_{i} \longrightarrow r_{i}+\sigma_{1 i} \dot{B}_{1 i}(t), \\
-\gamma_{i} \longrightarrow-\gamma_{i}+\sigma_{2 i} \dot{B}_{2 i}(t), \quad i=1,2, \ldots, n,
\end{gathered}
$$

where $B_{1 i}(t), B_{2 i}(t)$ are mutually independent Brownian motions and $\sigma_{1 i}, \sigma_{2 i}$ are positiveconstants representing the intensity of the white noises, respectively. Then the stochastic 
system takes the following form:

$$
\begin{gathered}
d x_{i}=\left[x_{i}\left(r_{i}-b_{i} x_{i}-e_{i} y_{i}\right)+\sum_{j=1}^{n} d_{i j}\left(x_{j}-\alpha_{i j} x_{i}\right)\right] d t+\sigma_{1 i} x_{i} d B_{1 i}(t), \\
d y_{i}=y_{i}\left(-\gamma_{i}-\delta_{i} y_{i}+\varepsilon_{i} x_{i}\right) d t+\sigma_{2 i} y_{i} d B_{2 i}(t), \quad i=1,2, \ldots, n
\end{gathered}
$$

Throughout this paper, we assume $d_{i j}$ are nonnegative constants, $\left(d_{i j}\right)$ is irreducible, and the parameters $r_{i}, \gamma_{i}, b_{i}, e_{i}, \delta_{i}, \varepsilon_{i}$ are positive constants.

In order to obtain better dynamic properties of the SDE (1.3), we will show that there exists a unique positive global solution with any initial positive value, and its $p$ th moment is bounded in Section 2. In the study of a population dynamics, permanence is a very important and interesting topic regarding the survival of populations in ecological system. In a deterministic system, it is usually solved by showing the global attractivity of the positive equilibrium of the system. But, as mentioned above, it is impossible to expect stochastic system (1.3) to tend to a steady state. So we attempt to investigate the stationary distribution of this system by Lyapunov functional technique. The stationary can be considered a weak stability, which appears as the solution is fluctuating in a neighborhood of the equilibrium point of the corresponding deterministic model. In Section 3, we will show if the white noise is small, there is a stationary distribution of SDE (1.3) and it has ergodic property. Existing results on dynamics in a patchy environment have largely been restricted to extinction analysis which means that the population system will survive or die out in the future due to the increased complexity of global analysis. In Section 4, we give the sufficient conditions for extinction. In Section 5, we make numerical simulation to conform our analytical results. Finally, for the completeness of the paper, we give an appendix containing some theories which will be used in previous sections.

The key method used in this paper is the analysis of Lyapunov functions $[6,13,14,16]$. We will also use the graph theory in Section 3 and some graph definitions can be found in the appendix.

Throughout this paper, unless otherwise specified, let $\left(\Omega,\left\{\mathcal{F}_{t}\right\}_{t \geq 0}, P\right)$ be a complete probability space with a filtration $\left\{\mathcal{F}_{t}\right\}_{t>0}$ satisfying the usual conditions (i.e., it is right continuous and $\Psi_{0}$ contains all $P$-null sets). Let $R_{+}^{2 n}$ denote the positive cone of $R^{2 n}$, namely, $R_{+}^{2 n}=\left\{\left(x_{1}, y_{1}, \ldots, x_{n}, y_{n}\right) \in R^{2 n}: x_{i}>0, y_{i}>0, i=1,2, \ldots, n\right\}$. For convenience and simplicity in the following discussion, denote $X(t)=\left(x_{1}(t), y_{1}(t), x_{2}(t), y_{2}(t), \ldots, x_{n}(t), y_{n}(t)\right)$. If $A$ is a vector or matrix, its transpose is denoted by $A^{T}$. If $A$ is a matrix, its trace norm is denoted by $|A|=\sqrt{\operatorname{trace}\left(A^{T} A\right)}$ whilst its operator norm is denoted by $\|A\|=\sup \{|A x|:|x|=1\}$.

\section{Positive and Global Solutions}

In order for a stochastic differential equation to have a unique global (i.e., no explosion at any finite time) solution, the coefficients of the equation are generally required to satisfy the linear growth condition and local Lipschitz condition (see [17]). However, the coefficients of SDE (1.3) do not satisfy the linear growth condition, though they are locally Lipschitz continuous, so the solution of SDE (1.3) may explode at a finite time. In this section, we will prove the solution of stochastic system (1.3) with any positive initial value is not only positive but also not exploive in infinity at any finite time. 
Theorem 2.1. For any given initial value $X(0) \in R_{+}^{2 n}$, there is a unique positive solution $X(t)$ of $S D E$ (1.3), and the solution will remain in $R_{+}^{2 n}$ with probability 1.

Proof. We define a $C^{2}$-function $V: R_{+}^{2 n} \rightarrow R_{+}$:

$$
V(X(t))=\sum_{i=1}^{n}\left[\varepsilon_{i}\left(x_{i}-1-\log x_{i}\right)+e_{i}\left(y_{i}-1-\log y_{i}\right)\right]
$$

Applying Itô's formula, we have

$$
\begin{aligned}
& L V=\sum_{i=1}^{n}\left(\varepsilon_{i}\left\{\left(x_{i}-1\right)\left[r_{i}-b_{i} x_{i}-e_{i} y_{i}+\sum_{j=1}^{n} d_{i j}\left(\frac{x_{j}}{x_{i}}-\alpha_{i j}\right)\right]+\frac{\sigma_{1 i}^{2}}{2}\right\}\right. \\
& \left.\quad+e_{i}\left[\left(y_{i}-1\right)\left(-\gamma_{i}-\delta_{i} y_{i}+\varepsilon_{i} x_{i}\right)+\frac{\sigma_{2 i}^{2}}{2}\right] d t\right) \\
& =\sum_{i=1}^{n}\left[-\varepsilon_{i} b_{i} x_{i}^{2}-e_{i} \delta_{i} y_{i}^{2}+\varepsilon_{i}\left(r_{i}-\sum_{j=1}^{n} d_{i j} \alpha_{i j}+b_{i}-e_{i}\right) x_{i}+\varepsilon_{i} \sum_{j=1}^{n} d_{i j} x_{j}\right. \\
& \left.\quad-\varepsilon_{i} \sum_{j=1}^{n} d_{i j} \frac{x_{j}}{x_{i}}+e_{i}\left(-\gamma_{i}+\delta_{i}+\varepsilon_{i}\right) y_{i}+\varepsilon_{i}\left(\sum_{j=1}^{n} d_{i j} \alpha_{i j}-r_{i}+\frac{\sigma_{1 i}^{2}}{2}\right)+e_{i}\left(\gamma_{i}+\frac{\sigma_{2 i}^{2}}{2}\right)\right] d t \\
& \leq \sum_{i=1}^{n}\left[\begin{array}{c}
-\varepsilon_{i} b_{i} x_{i}^{2}-e_{i} \delta_{i} y_{i}^{2}+\varepsilon_{i}\left(r_{i}-\sum_{j=1}^{n} d_{i j} \alpha_{i j}+b_{i}-e_{i}\right) x_{i}+\varepsilon_{i} \sum_{j=1}^{n} d_{i j} x_{j} \\
\left.+e_{i}\left(-\gamma_{i}+\delta_{i}+\varepsilon_{i}\right) y_{i}+\varepsilon_{i}\left(\sum_{j=1}^{n} d_{i j} \alpha_{i j}-r_{i}+\frac{\sigma_{1 i}^{2}}{2}\right)+e_{i}\left(\gamma_{i}+\frac{\sigma_{2 i}^{2}}{2}\right)\right] .
\end{array}\right.
\end{aligned}
$$

It is clear that the coefficient of quadratic term is negative, so we must be able to find a positive constant number $K$ that satisfies

$$
L V \leq K
$$

and $K$ is independent of $x_{i}, y_{i}$, and $t$. By the similar proof of Li and Mao [18, Theorem 2.1], we can obtain the desired assertion.

Theorem 2.1 shows that the solution of SDE (1.3) will remain in the positive cone $R_{+}^{2}$ with probability 1 . This nice property provides us with a great opportunity to discuss the $p$ th moment and stochastically ultimately boundedness of the solution. 
Definition 2.2 (see [18]). The solution $X(t)$ of SDE (1.3) is said to be stochastically ultimately bounded, if for any $\epsilon \in(0,1)$, there exists a positive constant $X(=X(\epsilon))$, such that for any initial value $X(0) \in R_{+}^{2 n}$, the solution $X(t)$ to (1.3) has the property that

$$
\limsup _{t \rightarrow \infty} P\{|x(t)|>x\}<\epsilon
$$

Lemma 2.3. For any given initial value $X(0) \in R_{+}^{2 n}$, there exist positive constants $\kappa(p), p_{i}$, and $q_{i}(i=1,2, \ldots, n)$, such that the solution $X(t)$ of SDE (1.3) has the following property:

$$
E\left[\sum_{i=1}^{n}\left(p_{i} x_{i}^{p}(t)+q_{i} y_{i}^{p}(t)\right)\right] \leq \kappa(p), \quad t \geq 0, p>1
$$

Proof. By Itô's formula and Young inequality, we compute

$$
\begin{aligned}
& d\left(\frac{1}{p} x_{i}^{p}\right)=x_{i}^{p-1} d x_{i}+\frac{p-1}{2} x_{i}^{p-2}\left(d x_{i}\right)^{2} \\
& =\left[-b_{i} x_{i}^{p+1}+\left(r_{i}+\frac{p-1}{2} \sigma_{1 i}^{2}-\sum_{j=1}^{n} d_{i j} \alpha_{i j}\right) x_{i}^{p}-e_{i} x_{i}^{p} y_{i}+\sum_{j=1}^{n} d_{i j} x_{i}^{p-1} x_{j}\right] d t \\
& +\sigma_{1 i} x_{i}^{p} d B_{1 i}(t) \\
& \leq\left[-b_{i} x_{i}^{p+1}+\left(r_{i}+\frac{p-1}{2} \sigma_{1 i}^{2}+\sum_{j=1}^{n} d_{i j} \alpha_{i j}\right) x_{i}^{p}+\sum_{j=1}^{n} d_{i j}\left(\frac{p-1}{p} x_{i}^{p}+\frac{1}{p} x_{j}^{p}\right)\right] d t \\
& +\sigma_{1 i} x_{i}^{p} d B_{1 i}(t) \\
& =\left[-b_{i} x_{i}^{p+1}+\left(r_{i}+\frac{p-1}{2} \sigma_{1 i}^{2}+\sum_{j=1}^{n} d_{i j} \alpha_{i j}+\frac{p-1}{p} \sum_{j=1}^{n} d_{i j}\right) x_{i}^{p}+\frac{1}{p} \sum_{j=1}^{n} d_{i j} x_{j}^{p}\right] d t \\
& +\sigma_{1 i} x_{i}^{p} d B_{1 i}(t) \text {, } \\
& d\left(\frac{1}{p} y_{i}^{p}\right)=y_{i}^{p-1} d y_{i}+\frac{p-1}{2} y_{i}^{p-2}\left(d y_{i}\right)^{2} \\
& =\left[-\delta_{i} y_{i}^{p+1}+\left(-\gamma_{i}+\frac{p-1}{2} \sigma_{2 i}^{2}\right) y_{i}^{p}+\varepsilon_{i} x_{i} y_{i}^{p}\right] d t+\sigma_{2 i} y_{i}^{p} d B_{2 i}(t) \\
& \leq\left[-\delta_{i} y_{i}^{p+1}+\left(-\gamma_{i}+\frac{p-1}{2} \sigma_{2 i}^{2}\right) y_{i}^{p}+\varepsilon_{i}\left(\frac{1}{p+1} k_{i}^{-p} x_{i}^{p+1}+\frac{p}{p+1} k_{i} y_{i}^{p+1}\right)\right] d t \\
& +\sigma_{2 i} y_{i}^{p} d B_{2 i}(t) \\
& =\left[\left(-\delta_{i}+\frac{p \varepsilon_{i} k_{i}}{p+1}\right) y_{i}^{p+1}+\left(-\gamma_{i}+\frac{p-1}{2} \sigma_{2 i}^{2}\right) y_{i}^{p}+\frac{\varepsilon_{i} k_{i}^{-p}}{p+1} x_{i}^{p+1}\right] d t+\sigma_{2 i} y_{i}^{p} d B_{2 i}(t),
\end{aligned}
$$


where $k_{i}(i=1,2, \ldots, n)$ are positive constants to be determined. Hence, for positive constants $p_{i}, q_{i}$, we have

$$
\begin{aligned}
& d\left[\sum_{i=1}^{n}\left(p_{i} x_{i}^{p}+q_{i} y_{i}^{p}\right)\right] \\
& \leq \sum_{i=1}^{n}\left\{-p p_{i} b_{i} x_{i}^{p+1}+p_{i}\left[p r_{i}+\frac{p(p-1)}{2} \sigma_{1 i}^{2}+\sum_{j=1}^{n} d_{i j}\left(p-1+p \alpha_{i j}\right)+\sum_{j=1}^{n} d_{j i} \frac{p_{j}}{p_{i}}\right] x_{i}^{p}\right. \\
& \left.+p q_{i}\left(-\delta_{i}+\frac{p \varepsilon_{i} k_{i}}{p+1}\right) y_{i}^{p+1}+p q_{i}\left(-\gamma_{i}+\frac{p-1}{2} \sigma_{2 i}^{2}\right) y_{i}^{p}+\frac{p q_{i} \varepsilon_{i} k_{i}^{-p}}{p+1} x_{i}^{p+1}\right\} d t \\
& +p \sum_{i=1}^{n} p_{i} \sigma_{1 i} x_{i}^{p} d B_{1 i}(t)+p \sum_{i=1}^{n} q_{i} \sigma_{2 i} y_{i}^{p} d B_{2 i}(t) \\
& =\sum_{i=1}^{n}\left\{-p\left(p_{i} b_{i}-\frac{q_{i} \varepsilon_{i} k_{i}^{-p}}{p+1}\right) x_{i}^{p+1}-p q_{i}\left(\delta_{i}-\frac{p \varepsilon_{i} k_{i}}{p+1}\right) y_{i}^{p+1}\right. \\
& +p_{i}\left[p r_{i}+\frac{p(p-1)}{2} \sigma_{1 i}^{2}+\sum_{j=1}^{n} d_{i j}\left(p-1+p \alpha_{i j}\right)+\sum_{j=1}^{n} d_{j i} \frac{p_{j}}{p_{i}}\right] x_{i}^{p} \\
& \left.+p q_{i}\left(-\gamma_{i}+\frac{p-1}{2} \sigma_{2 i}^{2}\right) y_{i}^{p}\right\} d t \\
& +p \sum_{i=1}^{n} p_{i} \sigma_{1 i} x_{i}^{p} d B_{1 i}(t)+p \sum_{i=1}^{n} q_{i} \sigma_{2 i} y_{i}^{p} d B_{2 i}(t) .
\end{aligned}
$$

Next, we claim that there exist $p_{i}>0, q_{i}>0$, and $k_{i}>0$ such that

$$
p_{i} b_{i}-\frac{q_{i} \varepsilon_{i} k_{i}^{-p}}{p+1}>0, \quad \delta_{i}-\frac{p \varepsilon_{i} k_{i}}{p+1}>0
$$

In fact, we only need $0<k_{i}<(p+1) \delta_{i} / p \varepsilon_{i}$ and $0<q_{i}=p_{i} / m$, where $m$ is a sufficiently large positive integer. Let 
Abstract and Applied Analysis

$$
\begin{gathered}
\alpha_{i}=: p r_{i}+\frac{p(p-1)}{2} \sigma_{1 i}^{2}+\sum_{j=1}^{n} d_{i j}\left(p-1+p \alpha_{i j}\right)+\sum_{j=1}^{n} d_{j i} \frac{p_{j}}{p_{i}} \\
\alpha_{i}^{\prime}=: p\left(-\gamma_{i}+\frac{p-1}{2} \sigma_{2 i}^{2}\right), \\
\beta_{i}=: p p_{i}^{-1 / p}\left(b_{i}-\frac{\varepsilon_{i} k_{i}^{-p}}{m(p+1)}\right), \\
\beta_{i}^{\prime}=: p q_{i}^{-1 / p}\left(\delta_{i}-\frac{p \varepsilon_{i} k_{i}}{p+1}\right), \\
\check{\alpha}=: \max \left\{\alpha_{1}, \alpha_{1}^{\prime}, \ldots, \alpha_{n}, \alpha_{n}^{\prime}\right\}, \\
\widehat{\beta}=: \min \left\{\beta_{1}, \beta_{1}^{\prime}, \ldots, \beta_{n}, \beta_{n}^{\prime}\right\} .
\end{gathered}
$$

It is clear that $\breve{\alpha}>0, \widehat{\beta}>0$. Then

$$
\begin{aligned}
d\left[\sum_{i=1}^{n}\left(p_{i} x_{i}^{p}(t)+q_{i} y_{i}^{p}(t)\right)\right] & \\
\leq & {\left[\check{\alpha} \sum_{i=1}^{n}\left(p_{i} x_{i}^{p}(t)+q_{i} y_{i}^{p}(t)\right)-\widehat{\beta} \sum_{i=1}^{n}\left(p_{i}^{(p+1) / p} x_{i}^{p+1}(t)+q_{i}^{(p+1) / p} y_{i}^{p+1}(t)\right)\right] d t } \\
& +p \sum_{i=1}^{n} p_{i} \sigma_{1 i} x_{i}^{p}(t) d B_{1 i}(t)+p \sum_{i=1}^{n} q_{i} \sigma_{2 i} y_{i}^{p}(t) d B_{2 i}(t) .
\end{aligned}
$$

Integrating it from 0 to $t$ and taking expectations of both sides, we obtain that

$$
\begin{aligned}
& \frac{d E\left[\sum_{i=1}^{n}\left(p_{i} x_{i}^{p}(t)+q_{i} y_{i}^{p}(t)\right)\right]}{d t} \\
& \leq\left\{\check{\alpha} E\left[\sum_{i=1}^{n}\left(p_{i} x_{i}^{p}(t)+q_{i} y_{i}^{p}(t)\right)\right]-\widehat{\beta} E\left[\sum_{i=1}^{n}\left(p_{i}^{(p+1) / p} x_{i}^{p+1}(t)+q_{i}^{(p+1) / p} y_{i}^{p+1}(t)\right)\right]\right\} \\
& \quad \leq\left\{\check{\alpha} E\left[\sum_{i=1}^{n}\left(p_{i} x_{i}^{p}(t)+q_{i} y_{i}^{p}(t)\right)\right]-(2 n)^{-1 / p} \widehat{\beta} E\left[\sum_{i=1}^{n}\left(p_{\mathrm{i}} x_{i}^{p}(t)+q_{i} y_{i}^{p}(t)\right)\right]^{(p+1) / p}\right\} \\
& =E\left[\sum_{i=1}^{n}\left(p_{i} x_{i}^{p}(t)+q_{i} y_{i}^{p}(t)\right)\right]\left\{\check{\alpha}-(2 n)^{-1 / p} \widehat{\beta} E\left[\sum_{i=1}^{n}\left(p_{i} x_{i}^{p}(t)+q_{i} y_{i}^{p}(t)\right)\right]^{1 / p}\right\} .
\end{aligned}
$$

Therefore, letting $z(t)=E\left[\sum_{i=1}^{n}\left(p_{i} x_{i}^{p}(t)+q_{i} y_{i}^{p}(t)\right)\right]$, we have

$$
\frac{d z(t)}{d t} \leq z(t)\left(\check{\alpha}-(2 n)^{-1 / p} \widehat{\beta} z^{1 / p}(t)\right) .
$$


Notice that the solution of equation

$$
\frac{d \bar{z}(t)}{d t}=\bar{z}(t)\left(\check{\alpha}-(2 n)^{-1 / p} \widehat{\beta} \bar{z}^{1 / p}(t)\right)
$$

obeys

$$
\bar{z}(t) \longrightarrow 2 n \cdot\left(\frac{\check{\alpha}}{\widehat{\hat{\beta}}}\right)^{p}, \quad \text { as } t \longrightarrow \infty
$$

By comparison theorem, we can get

$$
\limsup _{t \rightarrow \infty} E\left[\sum_{i=1}^{n}\left(p_{i} x_{i}^{p}(t)+q_{i} y_{i}^{p}(t)\right)\right] \leq 2 n \cdot\left(\frac{\check{\alpha}}{\widehat{\widehat{\beta}}}\right)^{p}:=L(p)
$$

which implies that there is a $T>0$, such that

$$
E\left[\sum_{i=1}^{n}\left(p_{i} x_{i}^{p}(t)+q_{i} y_{i}^{p}(t)\right)\right] \leq 2 L(p), \quad t>T .
$$

In addition $E\left[\sum_{i=1}^{n}\left(p_{i} x_{i}^{p}(t)+q_{i} y_{i}^{p}(t)\right)\right]$ is continuous, so we have

$$
E\left[\sum_{i=1}^{n}\left(p_{i} x_{i}^{p}(t)+q_{i} y_{i}^{p}(t)\right)\right] \leq C(p), \quad t \in[0, T]
$$

Let $\kappa(p)=\max \{2 L(p), C(p)\}$, then

$$
E\left[\sum_{i=1}^{n}\left(p_{i} x_{i}^{p}(t)+q_{i} y_{i}^{p}(t)\right)\right] \leq \kappa(p), \quad t \geq 0, p>1
$$

This completes the proof.

Theorem 2.4. The solutions of system (1.3) are stochastically ultimately bounded for any initial value $X(0) \in R_{+}^{2 n}$.

Proof. From Theorem 2.1, the solution $X(t)$ will remain in $R_{+}^{2 n}$ with probability 1 . Let $Q=\min \left\{p_{1}, q_{1}, p_{2}, q_{2}, \ldots, p_{n}, q_{n}\right\}$. Note that $|X(t)|=\left[\sum_{i=1}^{n}\left(x_{i}^{2}+y_{i}^{2}\right)\right]^{1 / 2}$ and $|X(t)|^{p} \leq$ $(2 n)^{p / 2}\left[\sum_{i=1}^{n}\left(x_{i}^{p}+y_{i}^{p}\right)\right]$. Therefore, we get

$$
E\left[\sum_{i=1}^{n}\left(p_{i} x_{i}^{p}(t)+q_{i} y_{i}^{p}(t)\right)\right] \geq Q E\left[\sum_{i=1}^{n}\left(x_{i}^{p}(t)+y_{i}^{p}(t)\right)\right] \geq(2 n)^{-p / 2} Q E|X(t)|^{p}
$$


and by (2.5) we have

$$
E|X(t)|^{p} \leq \frac{(2 n)^{p / 2}}{Q} \kappa(p)=: \widetilde{\kappa}(p)<+\infty .
$$

Applying the Chebyshev inequality yields the required assertion.

\section{Stationary Distribution}

In this section, we investigate there is a stationary distribution for SDE (1.3) instead of asymptotically stable equilibria. Before giving the main theorem, we first give a lemma (see $[2])$.

Lemma 3.1 (see [2]). Assume $\left(d_{i j}\right)$ is irreducible. If there exists $i$ such that $b_{i}>0$ or $\delta_{i}>0$, then, whenever a positive equilibrium $E^{*}$ exists for system (1.1), it is unique and globally asymptotically stable in the positive cone $R_{+}^{2 n}$.

In the section, we assume system (1.1) exists and the positive equilibrium $E^{*}=$ $\left(x_{1}^{*}, y_{1}^{*}, \ldots, x_{n}^{*}, y_{n}^{*}\right)$ satisfies the equation

$$
\begin{gathered}
x_{i}^{*}\left(r_{i}-b_{i} x_{i}^{*}-e_{i} y_{i}^{*}\right)+\sum_{j=1}^{n} d_{i j}\left(x_{j}^{*}-\alpha_{i j} x_{i}^{*}\right)=0, \\
y_{i}^{*}\left(-\gamma_{i}-\delta_{i} y_{i}^{*}+\varepsilon_{i} x_{i}^{*}\right)=0, \quad i=1,2, \ldots, n,
\end{gathered}
$$

where $x_{i}^{*}>0, y_{i}^{*}>0$.

We now state a theorem in which the graph theory will be used. Assume $c_{i}(i=$ $1,2, \ldots, n)$ defined as in Lemma A.1 are nonnegative constants and $A=\left(d_{i j} \varepsilon_{i} x_{j}^{*}\right)_{n \times n}(i=$ $1,2, \ldots, n)$.

Theorem 3.2. Assume $\delta<\min \left\{\min _{1 \leq i \leq n}\left\{c_{i} \varepsilon_{i} b_{i}\left(x_{i}^{*}\right)^{2}\right\}, \min _{1 \leq i \leq n}\left\{c_{i} e_{i} \delta_{i}\left(y_{i}^{*}\right)^{2}\right\}\right\}$. Then there is a stationary distribution $\mu(\cdot)$ for $\operatorname{SDE}(1.3)$ and it has ergodic property. Here $\left(x_{1}^{*}, y_{1}^{*}, \ldots, x_{n}^{*}, y_{n}^{*}\right)$ is the solution of (3.1), and $\delta=(1 / 2) \sum_{i=1}^{n}\left(c_{i} \varepsilon_{i} b_{i} x_{i}^{*} \sigma_{1 i}^{2}+c_{i} e_{i} \delta_{i} y_{i}^{*} \sigma_{2 i}^{2}\right)$.

Proof. Define $V: E_{l}=R_{+}^{2 n} \rightarrow R_{+}:$

$$
V(X(t))=\sum_{i=1}^{n}\left[\varepsilon_{i}\left(x_{i}-x_{i}^{*}-x_{i}^{*} \log \frac{x_{i}}{x_{i}^{*}}\right)+e_{i}\left(y_{i}-y_{i}^{*}-y_{i}^{*} \log \frac{y_{i}}{y_{i}^{*}}\right)\right] .
$$


Then

$$
\begin{array}{r}
L V=\sum_{i=1}^{n} c_{i}\left\{\varepsilon_{i}\left(x_{i}-x_{i}^{*}\right)\left[\left(r_{i}-b_{i} x_{i}-e_{i} y_{i}\right)+\sum_{j=1}^{n} d_{i j}\left(\frac{x_{j}}{x_{i}}-\alpha_{i j}\right)\right]\right. \\
\left.+e_{i}\left(y_{i}-y_{i}^{*}\right)\left(-\gamma_{i}-\delta_{i} y_{i}+\varepsilon_{i} x_{i}\right)+\frac{1}{2} \varepsilon_{i} x_{i}^{*} \sigma_{1 i}^{2}+\frac{1}{2} e_{i} y_{i}^{*} \sigma_{2 i}^{2}\right\} .
\end{array}
$$

By (3.1), we have

$$
\begin{gathered}
r_{i}=b_{i} x_{i}^{*}+e_{i} y_{i}^{*}-\sum_{j=1}^{n} d_{i j}\left(\frac{x_{j}^{*}}{x_{i}^{*}}-\alpha_{i j}\right), \\
-\gamma_{i}=\delta_{i} y_{i}^{*}-\varepsilon_{i} x_{i}^{*}, \quad i=1,2, \ldots, n .
\end{gathered}
$$

Substituting this into (3.3) gives

$$
\begin{aligned}
& L V=\sum_{i=1}^{n} c_{i}\left[-\varepsilon_{i} b_{i}\left(x_{i}-x_{i}^{*}\right)^{2}-e_{i} \delta_{i}\left(y_{i}-y_{i}^{*}\right)^{2}+\varepsilon_{i}\left(x_{i}-x_{i}^{*}\right) \sum_{j=1}^{n} d_{i j}\left(\frac{x_{j}}{x_{i}}-\frac{x_{j}^{*}}{x_{i}^{*}}\right)\right. \\
& \left.+\frac{1}{2} \varepsilon_{i} x_{i}^{*} \sigma_{1 i}^{2}+\frac{1}{2} e_{i} y_{i}^{*} \sigma_{2 i}^{2}\right] \\
& =\sum_{i=1}^{n} c_{i}\left[-\varepsilon_{i} b_{i}\left(x_{i}-x_{i}^{*}\right)^{2}-e_{i} \delta_{i}\left(y_{i}-y_{i}^{*}\right)^{2}+\frac{1}{2} \varepsilon_{i} x_{i}^{*} \sigma_{1 i}^{2}+\frac{1}{2} e_{i} y_{i}^{*} \sigma_{2 i}^{2}\right. \\
& \left.+\varepsilon_{i} \sum_{j=1}^{n} d_{i j} x_{j}^{*}\left(\frac{x_{j}}{x_{j}^{*}}-\frac{x_{i}}{x_{i}^{*}}-\frac{x_{i}^{*} x_{j}}{x_{i} x_{j}^{*}}+1\right)\right] \\
& =\sum_{i=1}^{n} c_{i}\left\{-\varepsilon_{i} b_{i}\left(x_{i}-x_{i}^{*}\right)^{2}-e_{i} \delta_{i}\left(y_{i}-y_{i}^{*}\right)^{2}+\frac{1}{2} \varepsilon_{i} x_{i}^{*} \sigma_{1 i}^{2}+\frac{1}{2} e_{i} y_{i}^{*} \sigma_{2 i}^{2}\right. \\
& \left.+\varepsilon_{i} \sum_{j=1}^{n} d_{i j} x_{j}^{*}\left[\left(-\frac{x_{i}}{x_{i}^{*}}+\log \frac{x_{i}}{x_{i}^{*}}\right)-\left(-\frac{x_{j}}{x_{j}^{*}}+\log \frac{x_{j}}{x_{j}^{*}}\right)+\left(1-\frac{x_{i}^{*} x_{j}}{x_{i} x_{j}^{*}}+\log \frac{x_{i}^{*} x_{j}}{x_{i} x_{j}^{*}}\right)\right]\right\} \\
& \leq \sum_{i=1}^{n} c_{i}\left\{-\varepsilon_{i} b_{i}\left(x_{i}-x_{i}^{*}\right)^{2}-e_{i} \delta_{i}\left(y_{i}-y_{i}^{*}\right)^{2}+\frac{1}{2} \varepsilon_{i} x_{i}^{*} \sigma_{1 i}^{2}+\frac{1}{2} e_{i} y_{i}^{*} \sigma_{2 i}^{2}\right. \\
& \left.+\varepsilon_{i} \sum_{j=1}^{n} d_{i j} x_{j}^{*}\left[\left(-\frac{x_{i}}{x_{i}^{*}}+\log \frac{x_{i}}{x_{i}^{*}}\right)-\left(-\frac{x_{j}}{x_{j}^{*}}+\log \frac{x_{j}}{x_{j}^{*}}\right)\right]\right\},
\end{aligned}
$$


Here we use the fact: $1-a+\log a \leq 0$, for $a>0$ with equality holding if and only if $a=1$. Since $\left(d_{i j}\right)$ is irreducible, $\varepsilon_{i}>0, x_{j}^{*}>0$, we know matrix $A=\left(d_{i j} \varepsilon_{i} x_{j}^{*}\right)$ is irreducible. Let $G_{i}\left(x_{i}\right)=-x_{i} / x_{i}^{*}+\log \left(x_{i} / x_{i}^{*}\right), G_{j}\left(x_{j}\right)=-x_{j} / x_{j}^{*}+\log \left(x_{j} / x_{j}^{*}\right)$, and by Lemma A.1, we have

$$
\sum_{i, j=1}^{n} c_{i}\left(d_{i j} \varepsilon_{i} x_{j}^{*}\right)\left(-\frac{x_{i}}{x_{i}^{*}}+\log \frac{x_{i}}{x_{i}^{*}}\right)=\sum_{i, j=1}^{n} c_{i}\left(d_{i j} \varepsilon_{i} x_{j}^{*}\right)\left(-\frac{x_{j}}{x_{j}^{*}}+\log \frac{x_{j}}{x_{j}^{*}}\right)
$$

which implies that

$$
L V \leq \sum_{i=1}^{n}\left[-c_{i} \varepsilon_{i} b_{i}\left(x_{i}-x_{i}^{*}\right)^{2}-c_{i} e_{i} \delta_{i}\left(y_{i}-y_{i}^{*}\right)^{2}\right]+\frac{1}{2} \sum_{i=1}^{n}\left(c_{i} \varepsilon_{i} x_{i}^{*} \sigma_{1 i}^{2}+c_{i} e_{i} y_{i}^{*} \sigma_{2 i}^{2}\right)
$$

The following proof of ergodicity is similar to Theorem 3.2 in [19]. Assume $\delta=$ $(1 / 2) \sum_{i=1}^{n}\left(c_{i} \varepsilon_{i} x_{i}^{*} \sigma_{1 i}^{2}+c_{i} e_{i} y_{i}^{*} \sigma_{2 i}^{2}\right)$, then

$$
L V \leq \sum_{i=1}^{n}\left[-c_{i} \varepsilon_{i} b_{i}\left(x_{i}-x_{i}^{*}\right)^{2}-c_{i} e_{i} \delta_{i}\left(y_{i}-y_{i}^{*}\right)^{2}\right]+\delta
$$

Note that $\delta<\min \left\{\min _{1 \leq i \leq n}\left\{c_{i} \varepsilon_{i} b_{i}\left(x_{i}^{*}\right)^{2}\right\}, \min _{1 \leq i \leq n}\left\{c_{i} e_{i} \delta_{i}\left(y_{i}^{*}\right)^{2}\right\}\right\}$, then the ellipsoid

$$
\sum_{i=1}^{n}\left[-c_{i} \varepsilon_{i} b_{i}\left(x_{i}-x_{i}^{*}\right)^{2}-c_{i} e_{i} \delta_{i}\left(y_{i}-y_{i}^{*}\right)^{2}\right]+\delta=0
$$

lies entirely in $R_{+}^{2 n}$. We can take $U$ to be a neighborhood of the ellipsoid with $\overline{\mathrm{U}} \subset E_{l}=R_{+}^{2 n}$, so for $X \in E_{l} \backslash U, L V \leq-N$ ( $N$ is a positive constant), which implies the condition (B.2) in Lemma A.2 is satisfied. Therefore, the solution $X(t)$ is recurrent in the domain $U$, which together with Remark A.3 and Lemma A.4 implies $X(t)$ is recurrent in any bounded domain $D \subset R_{+}^{2 n}$. Thus, for any $D$, there is

$$
M=\min \left\{\min _{1 \leq i \leq n}\left\{\sigma_{1 i}^{2} x_{i}^{2}\right\}, \min _{1 \leq i \leq n}\left\{\sigma_{2 i}^{2} y_{i}^{2}\right\},\left(x_{1}, y_{1}, \ldots, x_{n}, y_{n}\right) \in \bar{D}\right\}>0
$$

such that

$$
\sum_{i, j=1}^{n} a_{i j}(x) \xi_{i} \xi_{j}=\sum_{i=1}^{n}\left(\sigma_{1 i}^{2} x_{i}^{2} \xi_{i}^{2}+\sigma_{2 i}^{2} y_{i}^{2} \xi_{i}^{2}\right) \geq M\|\xi\|^{2}
$$

for all $\left(x_{1}, y_{1}, \ldots, x_{n}, y_{n}\right) \in \overline{\mathrm{U}}, \xi \in R^{n}$, which implies that condition (B.1) is also satisfied. Therefore, the stochastic system (1.3) has a stable stationary distribution $\mu(\cdot)$ and it is ergodic. 


\section{Extinction}

In this section, we will show that if the noise is sufficiently large, the solution to the associated SDE (1.3) will become extinct with probability 1, although the solution to the original equation (1.1) may be persistent. For example, recall a simple case, namely, the scalar logistic equation

$$
d N(t)=N(t)(a-b N(t)) d t, \quad t \geq 0,
$$

with initial value $N_{0}>0$. It is well known that, when $a>0, b>0$, the solution $N(t)$ is persistent, because

$$
\lim _{t \rightarrow \infty} N(t)=\frac{b}{a}
$$

However, consider its associated stochastic equation

$$
d N(t)=N(t)[(a-b N(t)) d t+\sigma d B(t)], \quad t \geq 0,
$$

where $\sigma>0$, then the solution to this stochastic equation will become extinct with probability 1 , that is to say, if $\sigma^{2}>2 a$,

$$
\lim _{t \rightarrow \infty} N(t)=0 \quad \text { a.s. }
$$

The following theorem reveals the important fact that the environmental noise may make the population extinct.

Theorem 4.1. For any given initial value $X(0) \in R_{+}^{2 n}$, the solution of the SDE (1.3) has the property that

$$
\limsup _{t \rightarrow \infty} \frac{\log \left(\sum_{i=1}^{n} x_{i}\right)}{t} \leq \check{l}_{1}-\frac{\widehat{\sigma}_{1}^{2}}{2} \text { a.s. }
$$

Here

$$
\check{l}_{1}=\max \left\{\max _{1 \leq i \leq n}\left\{r_{i}+\sum_{j=1}^{n} d_{j i}-\sum_{j=1}^{n} d_{i j} \alpha_{i j}\right\}, 0\right\}, \quad \frac{\widehat{\sigma}_{1}^{2}}{2}=\frac{1}{2 \sum_{i=1}^{n}\left(1 / \sigma_{1 i}^{2}\right)} .
$$

Particularly, if $\breve{l}_{1}-\widehat{\sigma}_{1}^{2} / 2<0$, then $\lim _{t \rightarrow \infty}\left(x_{1}(t), x_{2}(t), \ldots, x_{n}(t)\right)=0$ a.s.

Proof. Define

$$
V=\sum_{i=1}^{n} x_{i}
$$


By Itô's formula, we have

$$
\begin{aligned}
d V & =\sum_{i=1}^{n}\left[r_{i} x_{i}-b_{i} x_{i}^{2}-e_{i} x_{i} y_{i}+\sum_{j=1}^{n} d_{i j}\left(x_{j}-\alpha_{i j} x_{i}\right)\right] d t+\sum_{i=1}^{n} \sigma_{1 i} x_{i} d B_{1 i}(t) \\
& =\sum_{i=1}^{n}\left[-b_{i} x_{i}^{2}-e_{i} x_{i} y_{i}+\left(r_{i}+\sum_{j=1}^{n} d_{j i}-\sum_{j=1}^{n} d_{i j} \alpha_{i j}\right) x_{i}\right] d t+\sum_{i=1}^{n} \sigma_{1 i} x_{i} d B_{1 i}(t) .
\end{aligned}
$$

Thus we compute

$$
\begin{aligned}
d \log V= & \frac{1}{V} d V-\frac{1}{2 V^{2}}(d V)^{2} \\
= & \frac{1}{V} \sum_{i=1}^{n}\left[-b_{i} x_{i}^{2}-e_{i} x_{i} y_{i}+\left(r_{i}+\sum_{j=1}^{n} d_{j i}-\sum_{j=1}^{n} d_{i j} \alpha_{i j}\right) x_{i}\right] d t \\
& +\frac{1}{V} \sum_{i=1}^{n} \sigma_{1 i} x_{i} d B_{1 i}(t)-\frac{1}{2 V^{2}} \sum_{j=1}^{n} \sigma_{1 i}^{2} x_{i}^{2} d t
\end{aligned}
$$

Letting $\check{l}_{1}=\max \left\{\max _{1 \leq i \leq n}\left\{r_{i}+\sum_{j=1}^{n} d_{j i}-\sum_{j=1}^{n} d_{i j} \alpha_{i j}\right\}, 0\right\}$, we compute

$$
\frac{1}{V} \sum_{i=1}^{n}\left[-b_{i} x_{i}^{2}-e_{i} x_{i} y_{i}+\left(r_{i}+\sum_{j=1}^{n} d_{j i}-\sum_{j=1}^{n} d_{i j} \alpha_{i j}\right) x_{i}\right] \leq \frac{1}{V} \sum_{i=1}^{n}\left(r_{i}+\sum_{j=1}^{n} d_{j i}-\sum_{j=1}^{n} d_{i j} \alpha_{i j}\right) x_{i} \leq \check{l}_{1}
$$

By Cauchy inequality, we compute also

$$
-\frac{1}{2 V^{2}} \sum_{i=1}^{n} \sigma_{1 i}^{2} x_{i}^{2}=-\frac{\sum_{i=1}^{n}\left(\sigma_{1 i}^{2} x_{i}^{2}\right)}{2\left(\sum_{i=1}^{n} x_{i}\right)^{2}} \leq-\frac{\left(\sum_{i=1}^{n} x_{i}\right)^{2}}{2 \sum_{i=1}^{n}\left(1 / \sigma_{1 i}^{2}\right) \cdot\left(\sum_{i=1}^{n} x_{i}\right)^{2}}=-\frac{1}{2 \sum_{i=1}^{n}\left(1 / \sigma_{1 i}^{2}\right)}=:-\frac{\widehat{\sigma}_{1}^{2}}{2},
$$

where $\widehat{\sigma}_{1}^{2} / 2=1 / 2 \sum_{i=1}^{n}\left(1 / \sigma_{1 i}^{2}\right)$. Substituting these two inequalities into (4.9) yields

$$
d \log V \leq\left(\breve{l}_{1}-\frac{\widehat{\sigma}_{1}^{2}}{2}\right) d t+\frac{1}{V} \sum_{i=1}^{n} \sigma_{1 i} x_{i} d B_{1 i}(t)
$$

This implies

$$
\log V(X(t)) \leq \log V(X(0))+\int_{0}^{t}\left(\breve{l}_{1}-\frac{\widehat{\sigma}_{1}^{2}}{2}\right) d t+M(t)
$$


where $M(t)$ is a martingale defined by

$$
M(t)=\int_{0}^{t} \frac{1}{V} \sum_{i=1}^{n} \sigma_{1 i} x_{i}(s) d B_{1 i}(s)=\int_{0}^{t} \frac{\sum_{i=1}^{n} \sigma_{1 i} x_{i}(s) d B_{1 i}(s)}{\sum_{i=1}^{n} x_{i}(s)}
$$

with $M(0)=0$. The quadratic variation of this martingale is

$$
\langle M, M\rangle_{t}=\int_{0}^{t} \frac{\sum_{i=1}^{n} \sigma_{1 i}^{2} x_{i}^{2}(s)}{\left(\sum_{i=1}^{n} x_{i}(s)\right)^{2}} d s \leq \breve{\sigma}_{1}^{2} \int_{0}^{t} \frac{\sum_{i=1}^{n} x_{i}^{2}(s)}{\sum_{i=1}^{n} x_{i}^{2}(s)} d s=\check{\sigma}_{1}^{2} t,
$$

where $\check{\sigma}_{1}=\max _{1 \leq i \leq n}\left\{\sigma_{1 i}\right\}$. By the strong law of large numbers for martingale (see $[17,20]$ ), we have

$$
\lim _{t \rightarrow \infty} \frac{M(t)}{t}=0 \quad \text { a.s. }
$$

It finally follows from (4.13) by dividing $t$ on the both sides and then letting $t \rightarrow \infty$ that

$$
\limsup _{t \rightarrow \infty} \frac{\log V}{t} \leq \limsup _{t \rightarrow \infty} \frac{1}{t} \int_{0}^{t}\left(\check{l}_{1}-\frac{\widehat{\sigma}_{1}^{2}}{2}\right) d t=\check{l}_{1}-\frac{\widehat{\sigma}_{1}^{2}}{2} \quad \text { a.s. }
$$

which implies the required assertion.

Remark 4.2. Theorem 4.1 shows that if the condition $\breve{l}_{1}-\widehat{\sigma}_{1}^{2} / 2<0$ holds, that is, when the prey population is disturbed by large white noise, the species of prey will extinct.

Now we give the following theorem which describes the entire extinction.

Theorem 4.3. For any given initial value $X(0) \in R_{+}^{2 n}$, the solution of the SDE (1.3) has the property that

$$
\limsup _{t \rightarrow \infty} \frac{\log \left[\sum_{i=1}^{n}\left(\varepsilon_{i} x_{i}+e_{i} y_{i}\right)\right]}{t} \leq \check{l}-\frac{\widehat{\sigma}^{2}}{2} \quad \text { a.s. }
$$

Here

$$
\breve{l}=\max \left\{\max _{1 \leq i \leq n}\left\{r_{i}+\sum_{j=1}^{n}\left(d_{j i} \frac{\varepsilon_{j}}{\varepsilon_{i}}-d_{i j} \alpha_{i j}\right)\right\}, 0\right\}, \quad \frac{\widehat{\sigma}^{2}}{2}=\frac{1}{2 \sum_{i=1}^{n}\left(1 / \sigma_{1 i}^{2}+1 / \sigma_{2 i}^{2}\right)} .
$$

Particularly, if $\breve{l}-\widehat{\sigma}^{2} / 2<0$, then $\lim _{t \rightarrow \infty} X(t)=0$ a.s. 
Proof. The proof of the theorem is similar to Theorem 4.1, we only give the main proof procedure. Define

$$
V(X(t))=\sum_{i=1}^{n}\left(\varepsilon_{i} x_{i}+e_{i} y_{i}\right)
$$

Let $\breve{l}=: \max \left\{\max _{1 \leq i \leq n}\left\{r_{i}+\sum_{j=1}^{n} d_{j i}\left(\varepsilon_{j} / \varepsilon_{i}\right)-\sum_{j=1}^{n} d_{i j} \alpha_{i j}\right\}, 0\right\}$ and $\widehat{\sigma}^{2} / 2=1 / 2 \sum_{i=1}^{n}\left(1 / \sigma_{1 i}^{2}+1 / \sigma_{2 i}^{2}\right)$. By Itô's formula, we compute

$$
\begin{aligned}
d \log V \leq & \frac{1}{V} \sum_{i=1}^{n} \varepsilon_{i}\left(r_{i}+\sum_{j=1}^{n} d_{j i} \frac{\varepsilon_{j}}{\varepsilon_{i}}-\sum_{j=1}^{n} d_{i j} \alpha_{i j}\right) x_{i} d t-\frac{1}{2 V^{2}} \sum_{i=1}^{n}\left(\varepsilon_{i}^{2} \sigma_{1 i}^{2} x_{i}^{2}+e_{i}^{2} \sigma_{2 i}^{2} y_{i}^{2}\right) d t \\
& +\frac{1}{V}\left(\sum_{i=1}^{n} \varepsilon_{i} \sigma_{1 i} x_{i} d B_{1 i}(t)+\sum_{i=1}^{n} e_{i} \sigma_{2 i} y_{i} d B_{2 i}(t)\right) \\
\leq & \left(\check{l}-\frac{\widehat{\sigma}^{2}}{2}\right) d t+\frac{1}{V} \sum_{i=1}^{n}\left(\varepsilon_{i} \sigma_{1 i} x_{i} d B_{1 i}(t)+e_{i} \sigma_{2 i} y_{i} d B_{2 i}(t)\right) .
\end{aligned}
$$

The rest of the proof is similar to Theorem 4.1.

Remark 4.4. Theorem 4.3 states that when the prey and predator population are all disturbed by large white noise and the condition $\breve{l}-\widehat{\sigma}^{2} / 2<0$ holds, the two species will be extinct.

\section{Numerical Simulation}

In this section, in order to better study the effect of white noise in diffusion system, we assume $\alpha_{i j}=1, d_{i j}$ are nonnegative constants, $\left(d_{i j}\right)$ is irreducible $(i, j=1,2)$, and $d_{11}=d_{22}=1$. Consider the predator-prey system with $n=2$, that is,

$$
\begin{aligned}
& d x_{1}=\left[x_{1}\left(r_{1}-b_{1} x_{1}-e_{1} y_{1}\right)+d_{12}\left(x_{2}-x_{1}\right)\right] d t+\sigma_{11} x_{1} d B_{11}(t) \\
& d y_{1}=y_{1}\left(-\gamma_{1}-\delta_{1} y_{1}+\varepsilon_{1} x_{1}\right) d t+\sigma_{21} y_{1} d B_{21}(t) \\
& d x_{2}=\left[x_{2}\left(r_{2}-b_{2} x_{2}-e_{2} y_{2}\right)+d_{21}\left(x_{1}-x_{2}\right)\right] d t+\sigma_{12} x_{2} d B_{12}(t) \\
& d y_{2}=y_{2}\left(-\gamma_{2}-\delta_{2} y_{2}+\varepsilon_{2} x_{2}\right) d t+\sigma_{22} y_{2} d B_{22}(t) .
\end{aligned}
$$



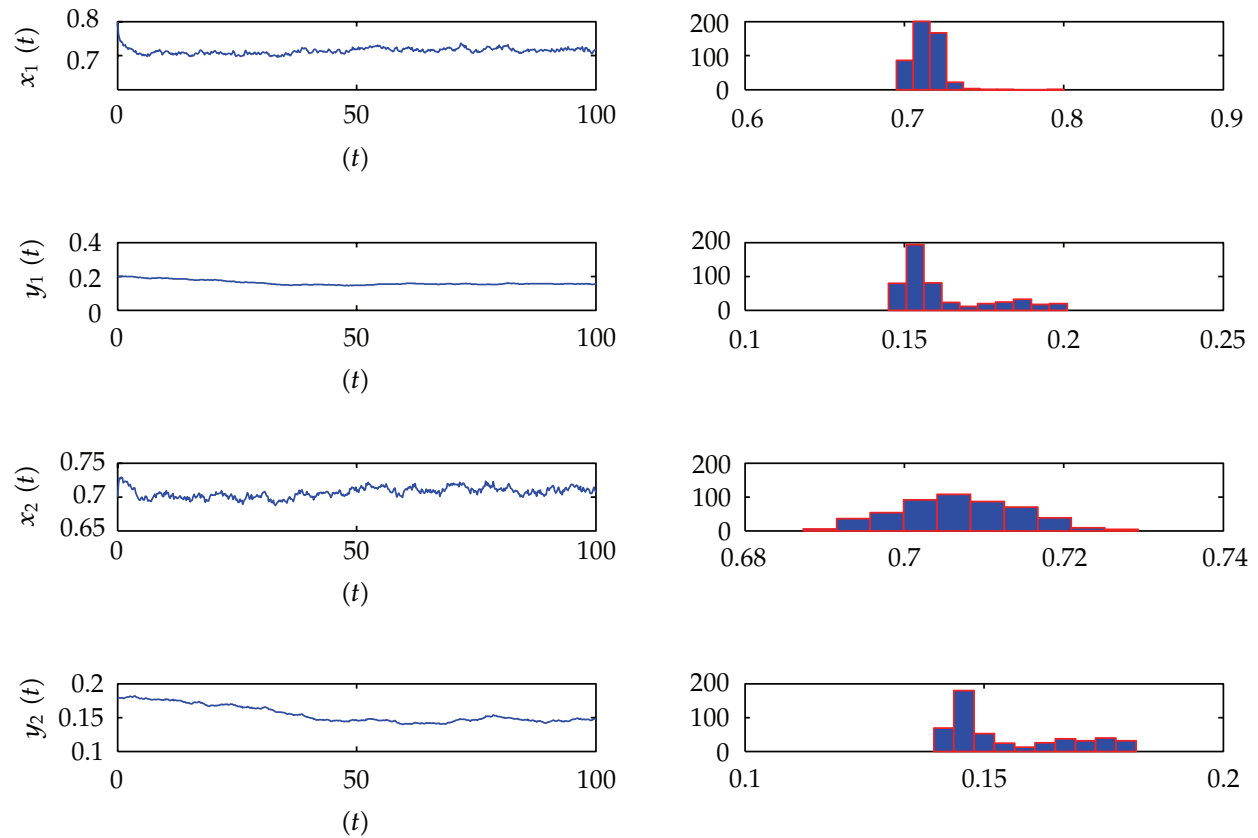

Figure 1: The solution of stochastic system (5.1) with small white noise and its histogram: $\sigma_{11}=\sigma_{12}=\sigma_{21}=$ $\sigma_{22}=0.01$.

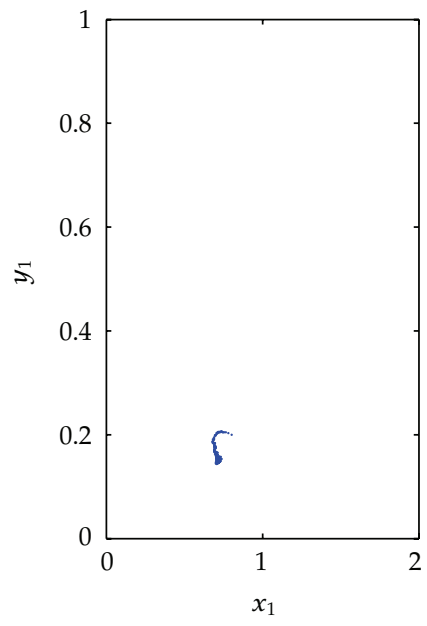

(a)

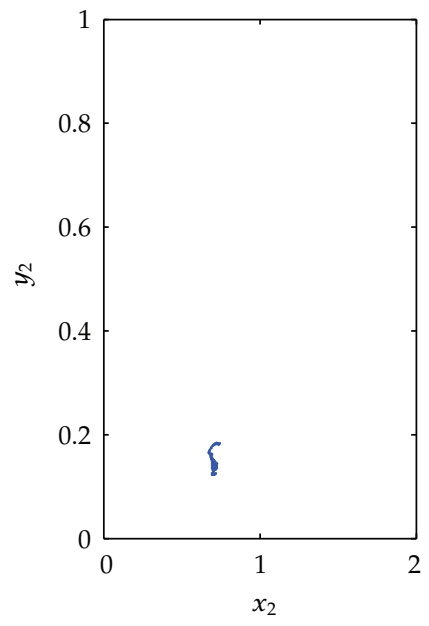

(b)

Figure 2: Population distribution scatter corresponding to Figure 1 . The scatter points around $\left(x_{1}^{*}, y_{1}^{*}\right) \doteq$ $(0.7174,0.1545)$ and $\left(x_{2}^{*}, y_{2}^{*}\right) \doteq(0.7107,0.1356)$, respectively. The system has a stationary distribution. 


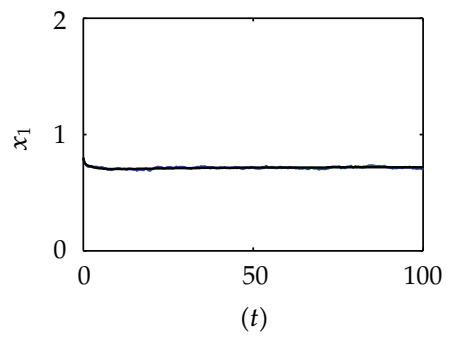

(a)

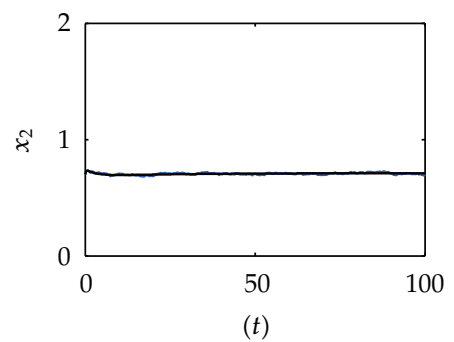

(c)

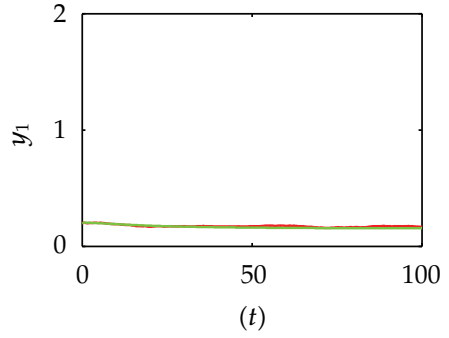

(b)

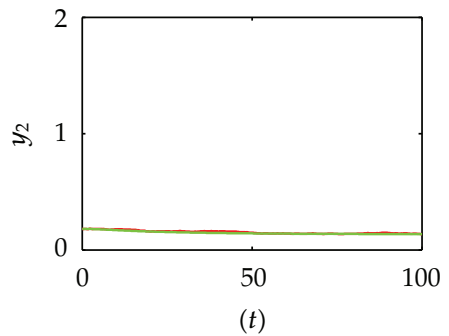

(d)

Figure 3: The solution of stochastic system compared to the deterministic system: $\sigma_{11}=\sigma_{12}=\sigma_{21}=\sigma_{22}=$ 0.01 . The blue and red lines represent the solutions of system (5.1), and the black and green lines represent the solutions of corresponding undisturbed system. These lines are almost coincident.
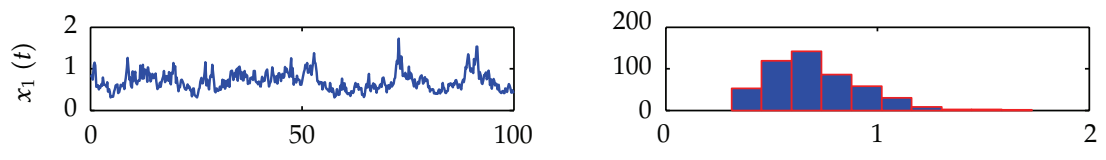

$(t)$
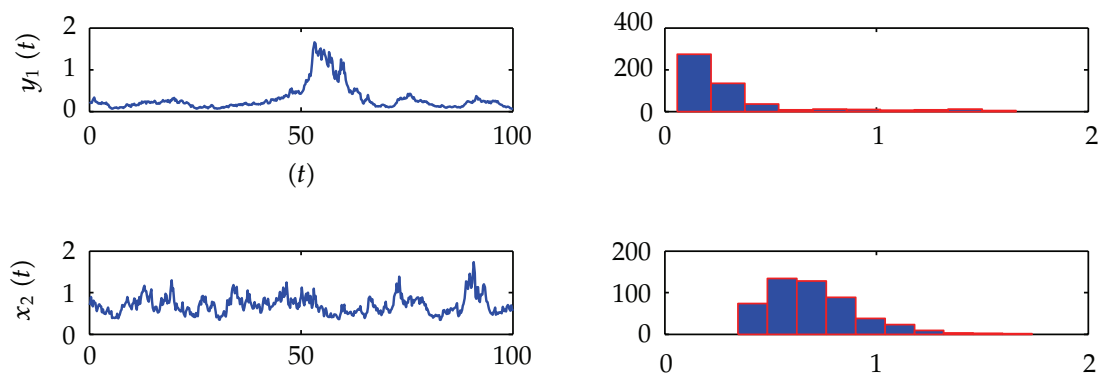

$(t)$
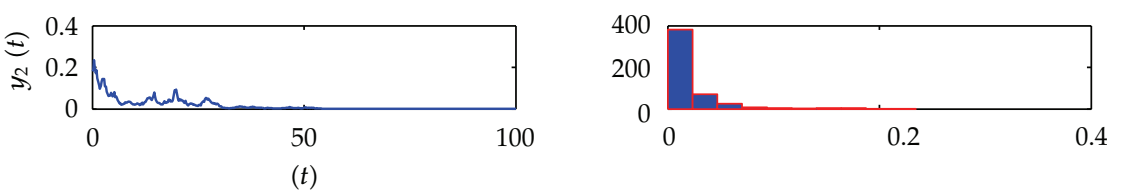

Figure 4: The solution of stochastic system with large white noise and its histogram: $\sigma_{11}=0.4, \sigma_{12}=$ $0.3, \sigma_{21}=0.3, \sigma_{22}=0.4$. The fluctuations on the left figures are more intense and histogram distribution is not concentrated comparing with Figure 1. 


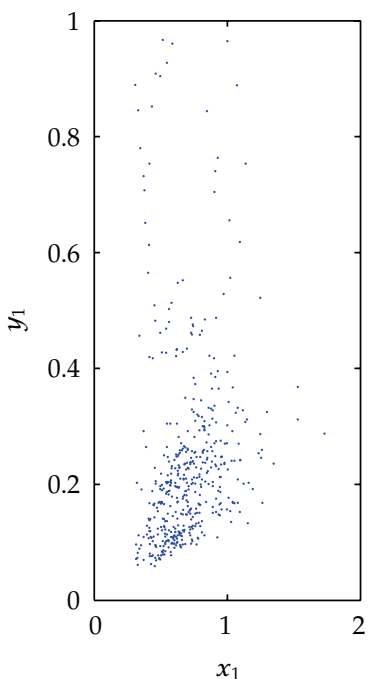

(a)

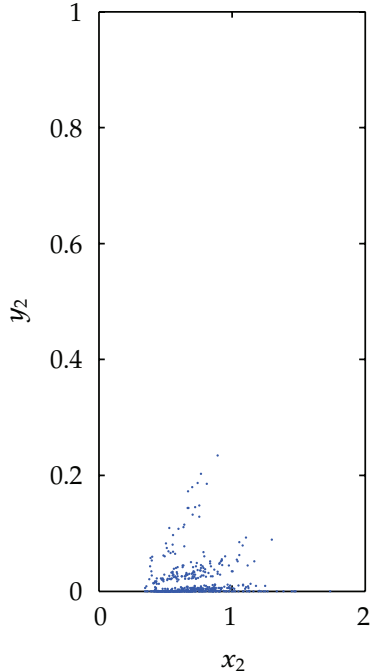

(b)

Figure 5: Population distribution scatter corresponding to Figure 4. The scatter is not around the equilibrium points of the corresponding deterministic system. There is not a stationary distribution in system (5.1).

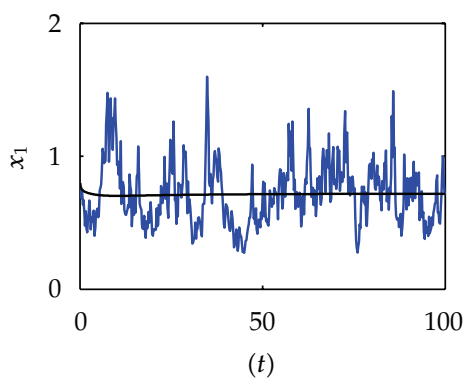

(a)

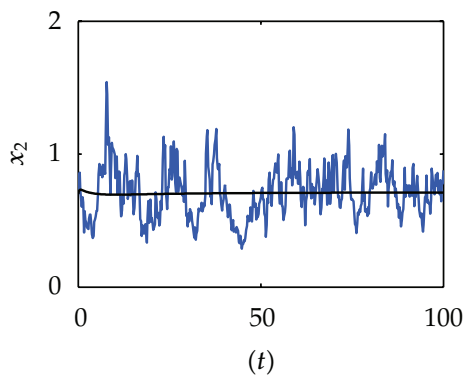

(c)

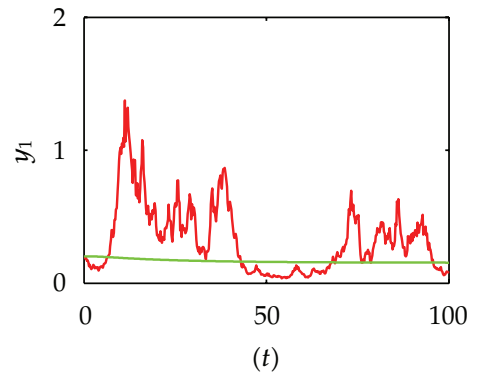

(b)

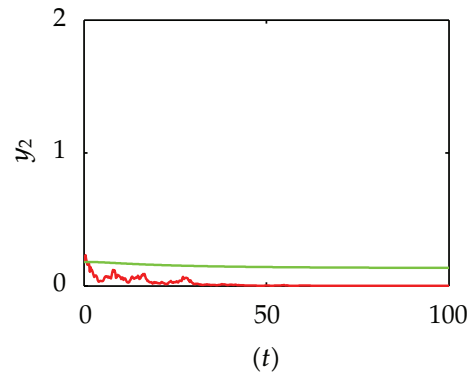

(d)

Figure 6: The solution of stochastic system compared to the deterministic system: $\sigma_{11}=0.4, \sigma_{12}=0.3, \sigma_{21}=$ $0.3, \sigma_{22}=0.4$. The predator population $y_{2}$ will die out although its corresponding deterministic system is globally stable. The blue and red lines represent the solutions of system (5.1), and the black and green lines represent the solutions of corresponding undisturb system. 


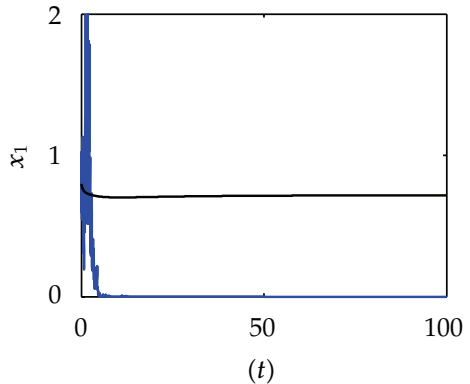

(a)

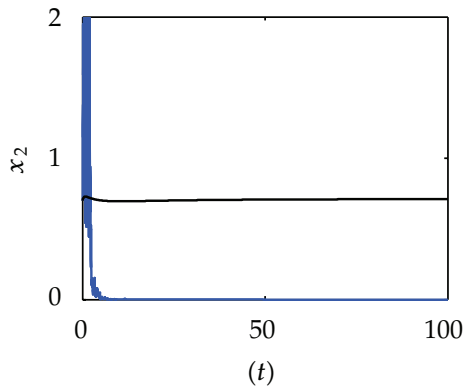

(c)

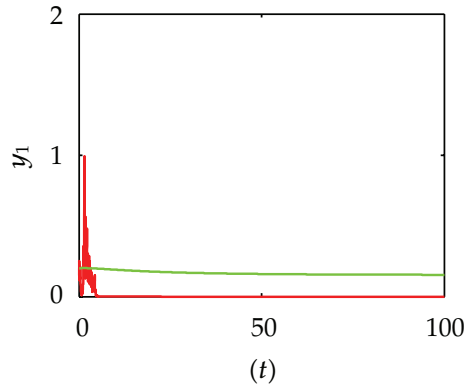

(b)

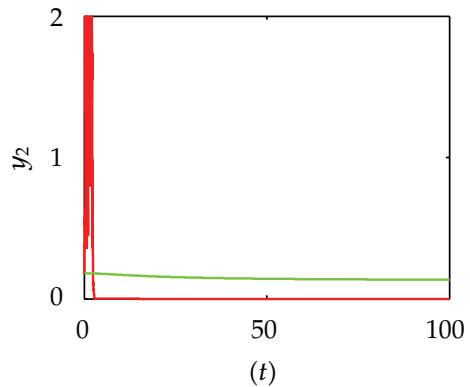

(d)

Figure 7: The solution of stochastic system compared to the deterministic system: $\sigma_{11}=1.4, \sigma_{12}=1.5, \sigma_{21}=$ $0.01, \sigma_{22}=0.01$. The prey population suffer the large white noise which leads to the prey and predator extinction. The blue and red lines represent the solutions of system (5.1), and the black and green lines represent the solutions of corresponding undisturb system.

In order to better study the previous results, we will numerically simulate the solution of (5.1). By the method mentioned in [21], we consider the following discretized equation:

$$
\begin{aligned}
& x_{1, k+1}=x_{1, k}+\left[x_{1, k}\left(r_{1}-b_{1} x_{1, k}-e_{1} y_{1, k}\right)+d_{12}\left(x_{2, k}-x_{1, k}\right)\right] h \\
& +\sigma_{11} x_{1, k} \sqrt{h} \xi_{1, k}+\frac{1}{2} \sigma_{11}^{2} x_{1, k}\left(h \xi_{1, k}^{2}-h\right), \\
& y_{1, k+1}=y_{1, k}+y_{1, k}\left(-\gamma_{1}-\delta_{1} y_{1, k}+\varepsilon_{1} x_{1, k}\right) h+\sigma_{21} y_{1, k} \sqrt{h} \eta_{1, k}+\frac{1}{2} \sigma_{21}^{2} y_{1, k}\left(h \eta_{1, k}^{2}-h\right), \\
& x_{2, k+1}=x_{2, k}+\left[x_{2, k}\left(r_{2}-b_{2} x_{2, k}-e_{2} y_{2, k}\right)+d_{21}\left(x_{1, k}-x_{2, k}\right)\right] h \\
& +\sigma_{12} x_{2, k} \sqrt{h} \xi_{2, k}+\frac{1}{2} \sigma_{12}^{2} x_{2, k}\left(h \xi_{2, k}^{2}-h\right), \\
& y_{2, k+1}=y_{2, k}+y_{2, k}\left(-\gamma_{2}-\delta_{2} y_{2, k}+\varepsilon_{2} x_{2, k}\right) h+\sigma_{22} y_{2, k} \sqrt{h} \eta_{2, k}+\frac{1}{2} \sigma_{22}^{2} y_{2, k}\left(h \eta_{2, k}^{2}-h\right) .
\end{aligned}
$$

Using the discretized equation and the help of Matlab software, we choose the appropriate parameters $r_{1}=0.4, r_{2}=0.3, b_{1}=0.5, b_{2}=0.4, e_{1}=e_{2}=0.2, d_{11}=$ $d_{22}=1, d_{12}=1.1, d_{21}=1.2, \gamma_{1}=\gamma_{2}=0.2, \delta_{1}=\delta_{2}=0.1, \varepsilon_{1}=\varepsilon_{2}=0.3$, the initial value $\left(x_{1}(0), y_{1}(0), x_{2}(0), y_{2}(0)\right)=(0.8,0.2,0.7,0.18)$, and time step $h=0.01$; then $E^{*}=$ 


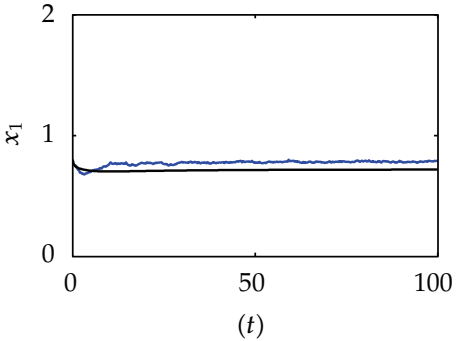

(a)

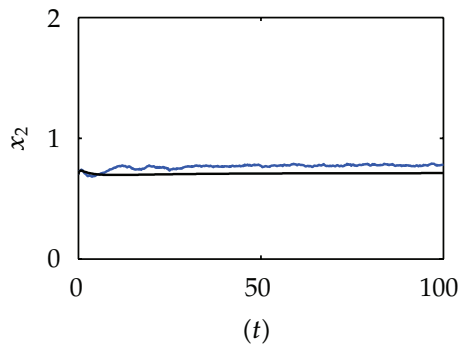

(c)

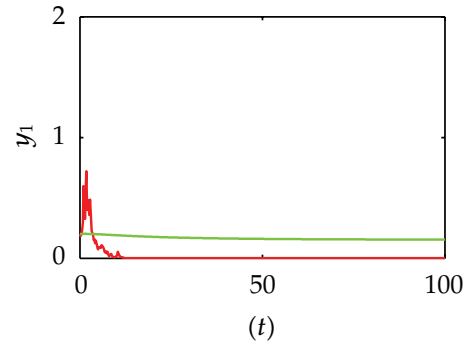

(b)

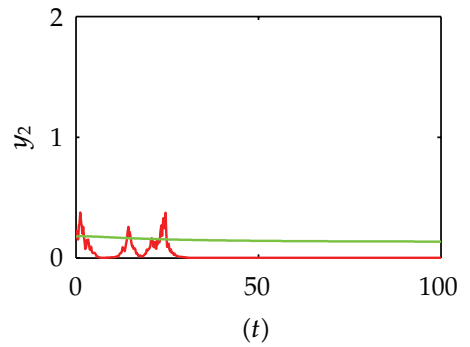

(d)

Figure 8: The solution of stochastic system compared to the deterministic system: $\sigma_{11}=\sigma_{12}=0.01, \sigma_{21}=$ $0.8, \sigma_{22}=0.7$. The predator population suffer the large white noise and then extinction. The blue and red lines represent the solutions of system (5.1), and the black and green lines represent the solutions of corresponding undisturb system.

$\left(x_{1}^{*}, y_{1}^{*}, x_{2}^{*}, y_{2}^{*}\right) \doteq(0.7174,0.1545,0.7107,0.1356)$. In order to better investigate the white noise, we divide its intensity into small, medium, and large three cases to study.

In Figures 1, 2, and 3, we choose $\sigma_{11}=\sigma_{12}=\sigma_{21}=\sigma_{22}=0.01$ and $c_{1} \doteq 0.86088, c_{2} \doteq 0.78177\left(c_{1}, c_{2}\right.$ satisfy Lemma A.1 and Theorem 3.2); then $\delta=(1 / 2) \sum_{i=1}^{2}\left(c_{i} \varepsilon_{i} b_{i} x_{i}^{*} \sigma_{1 i}^{2}+c_{i} e_{i} \delta_{i} y_{i}^{*} \sigma_{2 i}^{2}\right) \doteq 9.9 \times 10^{-6}$, and so the condition $\delta<$ $\min \left\{\min _{1 \leq i \leq 2}\left\{c_{i} \varepsilon_{i} b_{i}\left(x_{i}^{*}\right)^{2}\right\}, \min _{1 \leq i \leq 2}\left\{c_{i} e_{i} \delta_{i}\left(y_{i}^{*}\right)^{2}\right\}\right\} \doteq 4.11 \times 10^{-4}$ is also satisfied. Therefore, by Theorem 3.2, there is a stationary distribution (see the histogram on the right in Figure 1). From the left picture in Figure 1, we can see that the solution of system (5.1) is fluctuating in a small neighborhood. Moreover, from Figure 2, we find that almost all population distribution lies in the neighborhood, which can be imagined by a circular or elliptic region centered at $\left(x_{1}^{*}, y_{1}^{*}\right),\left(x_{2}^{*}, y_{2}^{*}\right)$ (see the scatter picture in Figure 2). Figure 3 shows that when the white noise is small, stochastic system imitates deterministic system and their curves are almost coincident. Hence, the solution of (5.1) is ergodicity, although there is no equilibrium of the stochastic system as the deterministic system. All of these imply system (5.1) is a stochastic stability.

Comparing with small white noise as in Figures 1, 2, and 3, we select the relatively large white noise $\sigma_{11}=0.4, \sigma_{12}=0.3, \sigma_{21}=0.3, \sigma_{22}=0.4, c_{1} \doteq 0.86088, c_{2} \doteq 0.78177$ in Figures 4 , 5 , and 6. We find that $\delta=0.0107>\min \left\{\min _{1 \leq i \leq 2}\left\{c_{i} \varepsilon_{i} b_{i}\left(x_{i}^{*}\right)^{2}\right\}, \min _{1 \leq i \leq 2}\left\{c_{i} e_{i} \delta_{i}\left(y_{i}^{*}\right)^{2}\right\}\right\}=2.875 \times$ $10^{-4}$, so the conditions of Theorem 3.2 are not satisfied; therefore, there is not a stationary distribution although the deterministic system is global asymptotic stability. The condition $\breve{l}-\widehat{\sigma}^{2} / 2>0$, that is, all extinction condition does not hold by Theorem 4.3 and the predator $y_{2}$ will die. From Figure 4, we see that the fluctuations on the left figures are more intense and 
histogram distribution is not concentrated comparing with Figure $1, y_{2}(t)$ close to the point 0 .

In Figure 7, we assume both the predator and the prey population suffered large white noise; we choose $\sigma_{11}=1.4, \sigma_{12}=1.5, \sigma_{21}=0.01, \sigma_{22}=0.01$, which satisfy the cases said in Theorem 4.1 , that is $\breve{l}_{1}-\widehat{\sigma}_{1}^{2} / 2 \doteq-0.0239<0$. As the case in Theorem 4.1 expected, the large white noise leads to the extinction of the prey which also leads to the extinction of the predator, so the solution of system (5.1) tends to zero.

In Figure 8, we choose the same parameters as in Figure 1, but change the value of $\sigma_{21}, \sigma_{22}$ to $\sigma_{21}=0.8, \sigma_{22}=0.7$, which do not meet the conditions for extinction of the two species as in Theorem 4.3. The predator population suffer the large white noises and then die out, but the prey will survive.

From these figures, we can get the following conclusions: when the white noise is small, system (5.1) imitates its deterministic system (see Figures 1, 2, and 3). But when the white noise is relatively large, it will bring more big deviation (see Figures 4, 5, and 6) even the extinction of the species (see Figures 6, 7, and 8), which will not happen in the deterministic system. However, when the intensity of the white noise on prey species is large, the predator and prey population will be extinct (see Figure 7). In real world, the large white noise may be bad weather, serious epidemic, which can be considered as the decisive factors responsible for the extinction of populations. Therefore, the study of stochastic model has great practical significance.

\section{Appendix}

In this section, we list some theories used in the previous sections.

\section{(1) Some Graph Theories [2, 22]}

A directed graph or digraph $\mathcal{G}=(V, E)$ contains a set $V=1,2, \ldots, n$ of vertices and a set $E$ of $\operatorname{arcs}(i, j)$ leading from initial vertex $i$ to terminal vertex $j$. In our convention, $a_{i j}>0$ if and only if there exists an arc from vertex $j$ to vertex $i$ in $\mathcal{G}$.

A digraph $\mathcal{G}$ is weighted if each arc $(j, i)$ is assigned a positive weight $a_{i j}$. Given a weighted digraph $(\mathcal{G}, A)$ with $n$ vertices, where $A=\left(a_{i j}\right)_{n \times n}$ is weight matrix, whose entry $a_{i j}$ equals the weight of arc $(j, i)$ if it exists, and 0 otherwise.

A digraph $(\mathcal{G}, A)$ is strongly connected if for any pair of distinct vertices, there exists a directed path from one to the other. A weighted digraph $(\mathcal{G}, A)$ is strongly connected iff the weight matrix $A$ is irreducible.

The Laplacian matrix of $(\mathcal{G}, A)$ is defined as

$$
L_{A}=\left[\begin{array}{cccc}
\sum_{i \neq 1} a_{1 i} & -a_{12} & \cdots & -a_{1 n} \\
-a_{21} & \sum_{i \neq 2} a_{2 i} & \cdots & -a_{2 n} \\
\vdots & \vdots & \ddots & \cdots \\
-a_{n 1} & a_{n 2} & \cdots & \sum_{i \neq n} a_{n i}
\end{array}\right] .
$$

Let $c_{i}$ denote the cofactor of the $i$ th diagonal element of $L_{A}$, which has the following property. 
Lemma A.1 (see [2]). Assume $n \geq 2$. If $(\mathcal{G}, A)$ is strongly connected. Then

(1) $c_{i}>0$ for $1 \leq i \leq n$,

(2) the following identity holds:

$$
\sum_{i, j=1}^{n} c_{i} a_{i j} G_{i}\left(x_{i}\right)=\sum_{i, j=1}^{n} c_{i} a_{i j} G_{j}\left(x_{j}\right)
$$

where $G_{i}\left(x_{i}\right)(1 \leq i \leq n)$ are arbitrary functions.

(2) Some Theories about the Stationary Distribution [23]

Let $X(t)$ be a homogeneous Markov Process in $E_{l}$ ( $E_{l}$ denotes $l$-space) described by the stochastic equation

$$
d X(t)=b(X) d t+\sum_{r=1}^{k} g_{r}(X) d B_{r}(t)
$$

The diffusion matrix is

$$
A(x)=\left(a_{i j}(x)\right), \quad a_{i j}(x)=\sum_{r=1}^{k} g_{r}^{i}(x) g_{r}^{j}(x) .
$$

Assumption B. There exists a bounded domain $U \subset E_{l}$ with regular boundary $\Gamma$, having the following properties:

(B.1) In the domain $U$ and some neighborhood thereof, the smallest eigenvalue of the diffusion matrix $A(x)$ is bounded away from zero.

(B.2) If $x \in E_{l} \backslash U$, the mean time $\tau$ at which a path issuing from $x$ reaches the set $U$ is finite and $\sup _{x \in K} E_{x} \tau<\infty$ for every compact subset $K \subset E_{l}$.

Lemma A.2 (see [23]). If Assumption B holds, then the Markov process X(t) has a stationary distribution $\mu(A)$. Let $f(\cdot)$ be a function integrable with respect to the measure $\mu$. Then

$$
P_{x}\left\{\lim _{T \rightarrow \infty} \frac{1}{T} \int_{0}^{T} f(X(t)) d t=\int_{E_{l}} f(x) \mu(d x)\right\}=1
$$

for all $x \in E_{l}$.

Remark A.3. Theorem 2.1 shows that there exists a unique positive solution $X(t)=$ $\left(x_{1}(t), y_{1}(t), \ldots x_{n}(t), y_{n}(t)\right)$ of SDE (1.3). Besides, from the proof of Theorem 2.1, we obtain

$$
L V \leq K
$$


Now define $\bar{V}=V+K$; then

$$
L \bar{V} \leq \bar{V}
$$

and we can get

$$
\bar{V}_{R}=\inf _{X \in R_{+}^{2 n} \backslash D_{m}} \bar{V}(X) \longrightarrow \infty \quad \text { as } m \longrightarrow \infty
$$

where $D_{m}=(1 / m, m) \times(1 / m, m) \times \cdots \times(1 / m, m)$. By [23] (Remark 2 of Theorem 4.1), we obtain the solution $X(t)$ is a homogeneous Markov process in $R_{+}^{2 n}$.

Lemma A.4 (see [23]). Let $X(t)$ be a regular temporally homogeneous Markov process in $E_{l}$. If $X(t)$ is recurrent relative to some bounded domain $U$, then it is recurrent relative to any nonempty domain in $E_{l}$.

\section{Acknowledgments}

The work was supported by the NSFC of China (no. 10971021), the NNSF of China (no. 11001032), the Ministry of Education of China (no. 109051), the Ph.D. Programs Foundation of Ministry of China (no. 200918) and Program for Changjiang Scholars and Innovative Research Team in University (PCSIRT).

\section{References}

[1] Y. Kuang and Y. Takeuchi, "Predator-prey dynamics in models of prey dispersal in two-patch environments," Mathematical Biosciences, vol. 120, no. 1, pp. 77-98, 1994.

[2] M. Y. Li and Z. Shuai, "Global-stability problem for coupled systems of differential equations on networks," Journal of Differential Equations, vol. 248, no. 1, pp. 1-20, 2010.

[3] E. Beretta, F. Solimano, and Y. Takeuchi, "Global stability and periodic orbits for two-patch predatorprey diffusion-delay models," Mathematical Biosciences, vol. 85, no. 2, pp. 153-183, 1987.

[4] H. I. Freedman and Y. Takeuchi, "Global stability and predator dynamics in a model of prey dispersal in a patchy environment," Nonlinear Analysis. Theory, Methods E Applications, vol. 13, no. 8, pp. 993 $1002,1989$.

[5] V. Padrón and M. C. Trevisan, "Environmentally induced dispersal under heterogeneous logistic growth," Mathematical Biosciences, vol. 199, no. 2, pp. 160-174, 2006.

[6] X. Mao, C. Yuan, and J. Zou, "Stochastic differential delay equations of population dynamics," Journal of Mathematical Analysis and Applications, vol. 304, no. 1, pp. 296-320, 2005.

[7] Z. Teng and L. Chen, "Permanence and extinction of periodic predator-prey systems in a patchy environment with delay," Nonlinear Analysis. Real World Applications, vol. 4, no. 2, pp. 335-364, 2003.

[8] W. Wendi and M. Zhien, "Asymptotic behavior of a predator-prey system with diffusion and delays," Journal of Mathematical Analysis and Applications, vol. 206, no. 1, pp. 191-204, 1997.

[9] R. Xu and L. Chen, "Persistence and stability for a two-species ratio-dependent predator-prey system with time delay in a two-patch environment," Computers $\mathcal{E}$ Mathematics with Applications, vol. 40, no. 4-5, pp. 577-588, 2000.

[10] J. Cui, "The effect of dispersal on permanence in a predator-prey population growth model," Computers \& Mathematics with Applications, vol. 44, no. 8-9, pp. 1085-1097, 2002.

[11] L. Zhang and Z. Teng, "Boundedness and permanence in a class of periodic time-dependent predatorprey system with prey dispersal and predator density-independence," Chaos, Solitons and Fractals, vol. 36, no. 3, pp. 729-739, 2008. 
[12] Z. Y. Lu and Y. Takeuchi, "Global asymptotic behavior in single-species discrete diffusion systems," Journal of Mathematical Biology, vol. 32, no. 1, pp. 67-77, 1993.

[13] C. Ji, D. Jiang, and N. Shi, "Analysis of a predator-prey model with modified Leslie-Gower and Holling-type II schemes with stochastic perturbation," Journal of Mathematical Analysis and Applications, vol. 359, no. 2, pp. 482-498, 2009.

[14] C. Ji, D. Jiang, and N. Shi, "A note on a predator-prey model with modified Leslie-Gower and Hollingtype II schemes with stochastic perturbation," Journal of Mathematical Analysis and Applications, vol. 377, no. 1, pp. 435-440, 2011.

[15] G. Cai and Y. Lin, "Stochastic analysis of predator-prey type ecosystems," Ecological Complexity, vol. 4, no. 4, pp. 242-249, 2007.

[16] X. Li, D. Jiang, and X. Mao, "Population dynamical behavior of Lotka-Volterra system under regime switching," Journal of Computational and Applied Mathematics, vol. 232, no. 2, pp. 427-448, 2009.

[17] X. Mao, Stochastic Differential Equations and Applications, Horwood, New York, NY, USA, 1997.

[18] X. Li and X. Mao, "Population dynamical behavior of non-autonomous Lotka-Volterra competitive system with random perturbation," Discrete and Continuous Dynamical Systems A, vol. 24, no. 2, pp. 523-545, 2009.

[19] C. Ji, D. Jiang, and H. Liu, "Existence, uniqueness and ergodicity of positive solution of mutualism system with stochastic perturbation," Mathematical Problems in Engineering, vol. 10, pp. 1155-1172, 2010.

[20] X. Mao and C. Yuan, Stochastic Differential Equations with Markovian Switching, Imperial College Press, London, UK, 2006.

[21] D. J. Higham, "An algorithmic introduction to numerical simulation of stochastic differential equations," SIAM Review, vol. 43, no. 3, pp. 525-546, 2001.

[22] H. Guo, M. Y. Li, and Z. Shuai, "A graph-theoretic approach to the method of global Lyapunov functions," Proceedings of the American Mathematical Society, vol. 136, no. 8, pp. 2793-2802, 2008.

[23] R. Z. Has'minskii, Stochastic Stability of Differential Equations, Sijthoff Noordhoff, Alphen aan den Rijn, The Netherlands, 1980. 


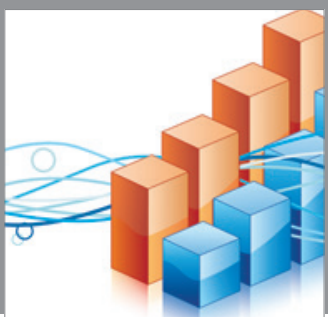

Advances in

Operations Research

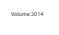

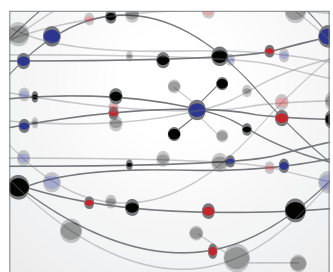

\section{The Scientific} World Journal
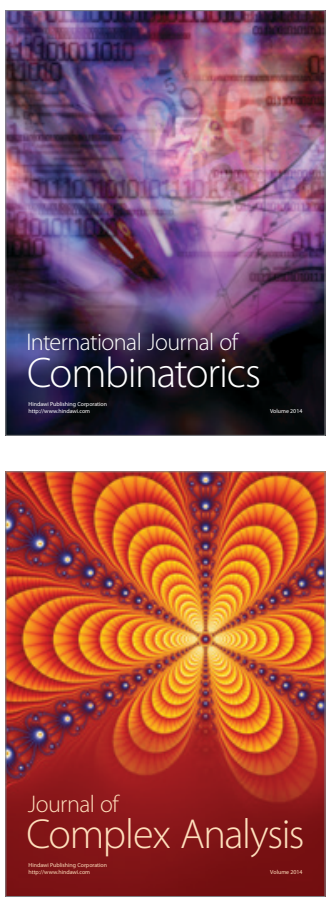

International Journal of

Mathematics and

Mathematical

Sciences
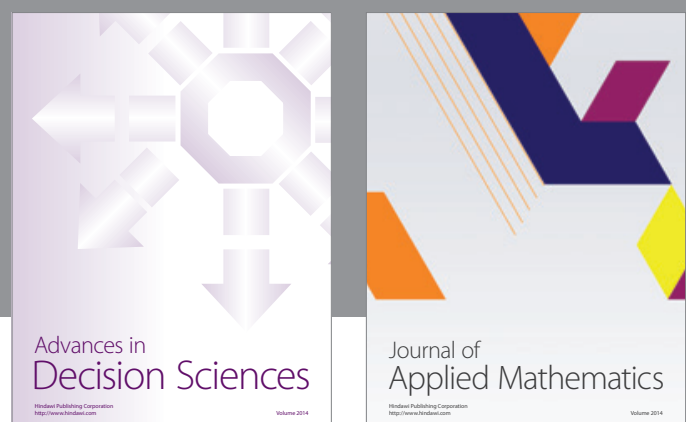

Journal of

Applied Mathematics
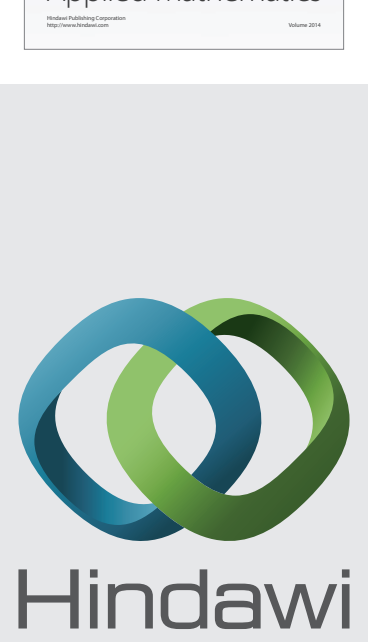

Submit your manuscripts at http://www.hindawi.com
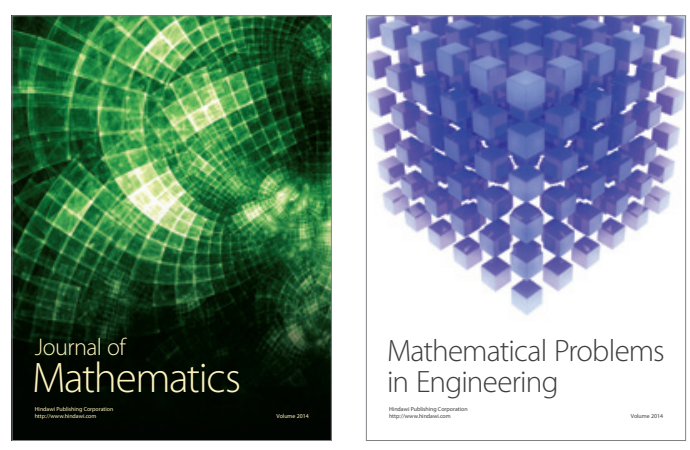

Mathematical Problems in Engineering
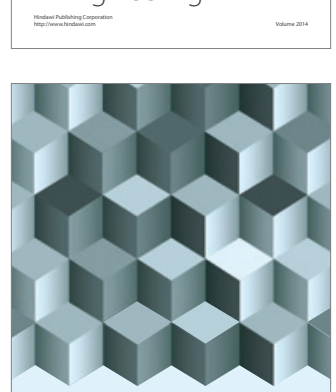

Journal of

Function Spaces
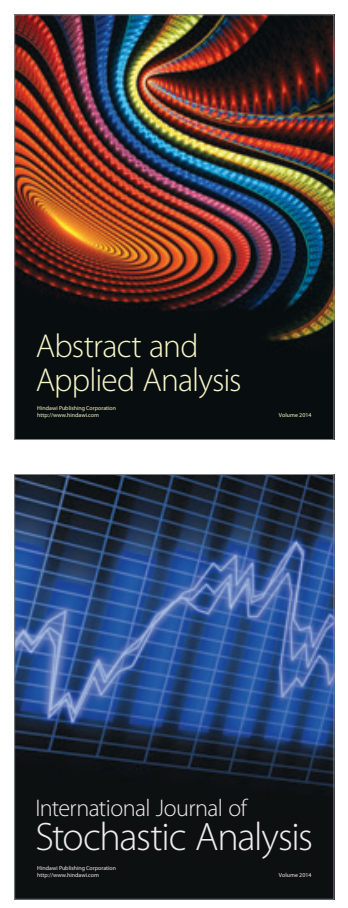

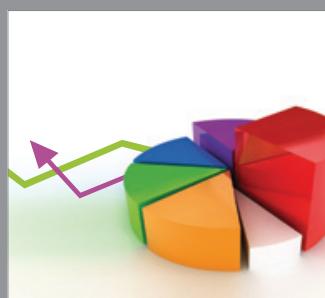

ournal of

Probability and Statistics

Promensencen
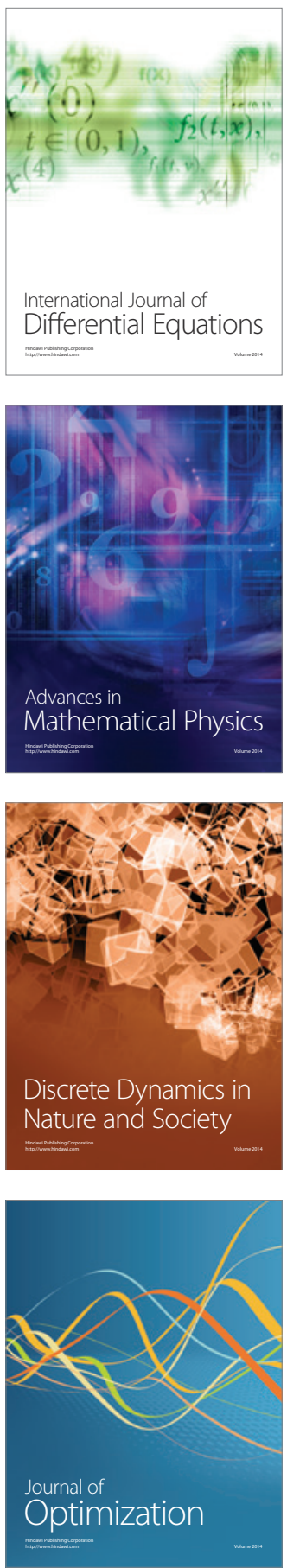